\title{
Estudo do comportamento das microcápsulas de polpa de tomate (Lycopersicum esculentum var. Carmen) imersas em azeite de oliva extra-virgem: interação e estabilidade do produto
}

\author{
Study of the behavior of tomato pulp microcapsules (Lycopersicum esculentum var. Carmen) \\ immersed in extra virgin olive oil: product interaction and stability
}

Estudio del comportamiento de microcápsulas de pulpa de tomate (Lycopersicum esculentum var. Carmen) sumergidas en aceite de oliva virgen extra: interacción y estabilidad del producto

\section{Resumo}

O objetivo deste trabalho foi estudar a interação entre os compostos bioativos da polpa de tomate encapsulado (técnica de gelificação iônica com 2\% (M2) e 5\% (M5) de alginato de sódio) e o azeite de oliva extra virgem, a influência da interação em relação à qualidade e a avaliar a estabilidade do produto final durante 60 dias de armazenamento. Foram feitas as análises de teor de licopeno, carotenoides, atividade antioxidante (ABTS), fenólicos totais, índice de peróxido, estabilidade oxidativa (Rancimat) e térmica (DSC). As análises foram realizadas em triplicata, aplicando o teste de Tukey ao nível de 5\% de significância. As formulações analisadas mantiveram-se estáveis durante o armazenamento, não houve diferença significativa nos teores de licopeno e carotenoides. A formulação M2 obteve menor interação do microencapsulado com o azeite (A2) em relação ao teor de fenólicos e a atividade antioxidante, mantendo assim os compostos fenólicos mais aprisionados. A inserção das microcápsulas no azeite de oliva extra virgem não alterou a sua qualidade mantendo-o na faixa exigida pela legislação ao índice de peróxido, além de não influenciar no tempo de indução à oxidação. A partir da análise térmica de DSC observou-se que as formulações M2 e M5 apresentaram picos de desidratação $\left(100-109^{\circ} \mathrm{C}\right)$ e degradação $\left(390-400^{\circ} \mathrm{C}\right)$ em uma mesma faixa, tendo assim características térmicas semelhantes. Desta forma, o fato da junção microcápsulas/azeite não interferiu na qualidade do azeite e agregou valor nutricional, evidenciando a interação dos compostos biativos, podendo ser modificado o tipo de encapsulado, ou do encapsulante e obter uma microcápsula com características diferentes de liberação dependendo do objetivo.

Palavras-chave: Licopeno; Gelificação iônica; Estabilidade, Antioxidante.

\footnotetext{
Abstract

The objective of this work was to microencapsulate the tomato pulp by the ionic gelation technique with $2 \%$ (M2) and $5 \%$ (M5) sodium alginate, immerse in extra virgin olive oil, characterize and evaluate the stability of this product during 60 days of storage. The analysis of lycopene content, carotenoids, antioxidant activity (ABTS), total phenolics, peroxide index, oxidative stability (Rancimat) and thermal (DSC) were performed in triplícate. The Tukey test was
} 
applied at the $5 \%$ level of significance. The formulations analyzed remained stable during storage, there was no significant difference in the levels of lycopene and carotenoids. The formulation M2 obtained minor interaction between the microencapsulated and olive oil (A2) about the phenolic content and antioxidant activity, in this way kept phenolic compounds trapped. The insertion of microcapsules in extra virgin olive oil has not quality decreased. The range required by the legislation regarding peroxide index was maintained and the oxidation induction time was not influenced. The DSC thermal analysis for the formulations M2 and M5 obtained peaks of dehydration $\left(100-109^{\circ} \mathrm{C}\right)$ and degradation $\left(390-400^{\circ} \mathrm{C}\right)$ in the same range, showing thermal characteristics similar. The microcapsule/oil complex maintained the quality of the oil and added nutritional value. The type of encapsulated, or encapsulant, can be modified and a microcapsule can be obtained with different release characteristics.

Keywords: Lycopene; Ionic gelation; Stability antioxidant.

\section{Resumen}

El objetivo de este trabajo fue microencapsular la pulpa de tomate mediante la técnica de gelificación iónica con alginato de sodio al 2\% (M2) y al 5\% (M5), adicionándolos en aceite de oliva extra virgen, caracterizando y evaluando la estabilidad de este producto durante 60 días de almacenamiento. Se realizaron análisis contenido de licopeno, carotenoides, actividad antioxidante (ABTS), fenoles totales, índice de peróxido, estabilidad oxidativa (Rancimat) y térmica (DSC). Los análisis se realizaron por triplicado, aplicando la prueba de Tukey al nivel de significancia del 5\%. Las formulaciones analizadas se mantuvieron estables durante el almacenamiento, no hubo diferencia significativa en los niveles de licopeno y carotenoides. La formulación M2 obtuvo una menor interacción entre el microencapsulado y el aceite de oliva (A2) en relación al contenido fenólico y la actividad antioxidante, manteniendo así los compuestos fenólicos más aprisionados. La inserción de microcápsulas en aceite de oliva extra virgen no modificó su calidad, manteniéndolo en el rango exigido por la legislación en materia de índice de peróxidos, además de no influir en el tiempo de inducción de oxidación. A partir del análisis térmico DSC se observó que las formulaciones M2 y M5 mostraron picos de deshidratación $\left(100-109^{\circ} \mathrm{C}\right)$ y degradación $\left(390-400^{\circ} \mathrm{C}\right)$ en el mismo rango, por lo que tenían características térmicas similares. Así, el hecho de que la unión microcápsula / aceite no interfiera con la calidad del aceite y también agregua valor nutricional, resalta la relevancia de su inserción, y el tipo de encapsulado, o encapsulante, se puede modificar y obtener una microcápsula con diferente liberación y características.

Palabras clave: Licopeno; Gelificación iónica; Estabilidad antioxidante.

\section{Introdução}

O tomate (Solanum lycopersicum) é uma fruta economicamente importante, amplamente consumida na forma in natura e processada. É um produto rico em componentes tais como açúcar, vitaminas e carotenoides com grande destaque para o licopeno, seu principal componente bioativo, responsável pela cor vermelha da sua polpa (Rocha, 2017). O licopeno apresenta propriedades antioxidantes de grande relevância, agindo na retirada do oxigênio singleto (forma mais reativa do oxigênio) e na eliminação de radicais livres (Di Mascio et al., 1991). À vista disso, apresenta um efeito positivo à saúde humana, onde seu consumo tem sido relacionado à redução do risco do desenvolvimento de certas doenças, como câncer de próstata, cervical e doença cardíaca coronária, além de atuar no metabolismo do colesterol, agir como anti-inflamatório e inibir a angiogênese tumoral (Costa-Rodrigues et al., 2018; Clinton, 1998; Palozza et al., 2012; Zu et al., 2014). Entretanto, em virtude da presença de um conjunto de ligações duplas em sua estrutura, o licopeno é altamente instável a fatores como oxigênio, luz, calor e umidade, afetando seu processamento e estocagem, uma vez que pode sofrer oxidação, que acarreta na perda de sua coloração e atividade funcional (Rodriguez-Amaya, 2001; Ranveer et al., 2015). Nesse contexto, alternativas tecnológicas têm sido estudadas a fim de melhorar a conservação deste composto. Estudos têm verificado que a encapsulação amplia a estabilidade do licopeno quando exposto a condições adversas (Goula; Adamopoulos, 2012; Choudhari et al., 2012; Ha et al., 2015; Rocha et al., 2012).

A encapsulação é uma técnica que consiste na retenção ou liberação controlada de um composto de interesse no interior de uma fina camada de material protetor (biopolímero), proporcionando barreira contra interações químicas e ambientais, ou a liberação desejada (Carmo et al., 2015). O encapsulamento facilita a manipulação, reduz o volume e aumenta a vida de prateleira dos antioxidantes naturais como o licopeno, melhorando sua estabilidade e promovendo a liberação gradual. Auxilia no manuseio de produtos líquidos ou gasosos, que a partir dessa técnica permanecem na forma sólida (Fang; 
Bhandari, 2010). Além disso, possibilita a "compartimentação" de materiais que reagem entre si; disfarça gosto de componentes desagradáveis, suavizando seu sabor; protege contra a luz, água e calor; além de aumentar a vida útil e proteger compostos de valor nutricional, evitando a oxidação (Fávaro-Trindade et al., 2008; Mirzaei, et al., 2012). Desta forma o objetivo deste trabalho foi estudar a interação dos compostos bioativos encapsulados por gelificação iônica e imersos em azeite de oliva entra virgem e a estabilidade do produto durante 60 dias de vida de prateleira.

\section{Metodologia}

Este trabalho foi desenvolvido no Laboratório de Análise de Alimentos pertencente ao departamento de Tecnologia de Alimentos (DTA) da Universidade Federal de Sergipe (UFS), localizada na cidade de São Cristóvão, Sergipe, Brasil. Os tomates tipo "Carmem" e o azeite de oliva extra virgem foram obtidos em um supermercado da cidade de Aracaju/SE.

\subsection{Obtenção da polpa do tomate e processo de gelificação iônica}

Primeiramente os tomates foram sanitizados em uma solução de hipoclorito de sódio (200 ppm) por 15 minutos e posteriormente imersos em água filtrada (5 min.), para retirar o excesso de sanitizante. Após a sanitização, foram retiradas as sementes e os tomates foram triturados em um liquidificador industrial e concentrados por meio de tratamento térmico $\left(80^{\circ} \mathrm{C} / 7 \mathrm{~min}.\right)$.

Foram desenvolvidas duas formulações de microcápsulas, com o percentual do agente encapsulante (alginato de sódio) e a quantidade de água diferentes. A massa da polpa de tomate obtida serviu de base de cálculo para a quantidade de água e encapsulante utilizados:

Formulação M2: $2 \%$ de alginato de sódio $(\mathrm{m} / \mathrm{m})$, polpa de tomate concentrada e água filtrada da proporção de $1: 2$ (massa de polpa: volume de água);

Formulação M5: 5\% de alginato de sódio $(\mathrm{m} / \mathrm{m})$, polpa de tomate concentrada e água filtrada da proporção de 1:3 (massa de polpa: volume de água).

Para o processo de gelificação iônica foi utilizada a metodologia de gotejamento descrita por Passos et al.(2021).Após a gelificação as microcápsulas foram drenadas e secas em secador elétrico $\left(87^{\circ} \mathrm{C} / 15 \mathrm{~min}\right.$.).

\subsection{Incorporação das microcápsulas de tomate ao Azeite de Oliva extra virgem}

As microcápsulas foram incorporadas ao azeite de oliva extra virgem na proporção de 1:2 (massa de microcápsulas: volume de azeite) e desta forma foram gerados dois grupos amostrais, grupo 1 (AM2), formado pelas microcápsulas da formulação M2 e o grupo 2 (AM5), formado pelas microcápsulas da formulação M5. Estas amostras foram condicionadas em garrafas de $100 \mathrm{~mL}$ transparente devidamente sanitizadas e lacradas, a uma temperatura de $27^{\circ} \mathrm{C}$ por 60 dias (Passos et al., 2021).

\subsection{Caracterização física e físico-química}

Para quantificar os carotenoides totais, foi utilizada a metodologia proposta por Lichtenthaler, (1987) e para a realização da análise do teor de licopeno foi utilizada a metodologia proposta por Rodriguez-amaya, (2001).

Para determinação dos compostos fenólicos e atividade antioxidante, inicialmente foi realizada uma extração: foi pesado $5 \mathrm{~g}$ de cada amostra e inserido em um Erlenmeyer de $250 \mathrm{~mL}$ juntamente com $50 \mathrm{~mL}$ de álcool etílico PA, posteriormente ao abrigo de luz foi agitado por agitação magnética por uma hora, após este procedimento o extrato foi filtrado e armazenado em vaso âmbar de $100 \mathrm{~mL}$. Este extrato seguiu para as análises de fenólicos totais pelo método de Folin- 
Ciocalteu, seguindo as adaptações de Swain e Hill (1959) e atividade antioxidante pelo método de ABTS conforme a metodologia descrita por Boroski et al. (2015).

As microcápsulas foram avaliadas quanto ao tamanho, utilizando um paquímetro digital Pantec com a escala de 0 a 150 $\mathrm{mm}$, e quanto ao peso, foram pesadas em balança analítica. Este procedimento foi realizado em quintuplicata.

A análise de calorimetria diferencial de varredura (DSC) foi realizada em equipamento TA- 60WS DSC-60 da Shimadzu. Para cada amostra de microcápsula (aproximadamente $3 \mathrm{mg}$ ), foram aquecidos em recipientes de alumínio a uma taxa de $10{ }^{\circ} \mathrm{C}$.min- 1 entre 25 e $550{ }^{\circ} \mathrm{C}$, com um fluxo de nitrogênio de $40 \mathrm{~mL} / \mathrm{min}$.

A determinação do índice de peróxido para os azeites, foi realizada segundo a método $\mathrm{Cd}$ 8-53 da American Oil Chemists' Society- AOCS (2003) e a estabilidade oxidativa foi realizada em um equipamento Rancimat, modelo 743, da Metrohm, seguindo a metodologia descrita no método Cd12b-92 da AOCS (2003). Os ensaios de estabilidade oxidativa foram realizados, em duplicata e os resultados foram expressos em horas.

\subsection{Análise Estatística}

Foi realizado um delineamento experimental, com três repetições de cada experimento. Os dados foram expressos como médias. Aplicou-se o teste de Tukey a 5\% de significância, para deteç̧ão de diferenças entre as médias dos diferentes tratamentos, por meio do software de estatística ASSISTAT versão 7.7 beta (Silva; Azevedo, 2009).

\section{Resultados e Discussão}

\subsection{Caracterização da polpa de tomate:}

As análises realizadas para a caracterização da polpa de tomate concentrada foram: teor de licopeno $(\mu \mathrm{g} / \mathrm{g})$, carotenoides totais ( $\mu \mathrm{g} / \mathrm{g}$ ), atividade antioxidante (mmol Trolox/g de amostra) e compostos fenólicos (mgEAG/g de amostra). Os resultados destas análises estão apresentados na Tabela 2.

Tabela 2: Análises de caracterização da polpa de tomate concentrada

\begin{tabular}{cc}
\hline Análises & Resultados \\
\hline Teor de Licopeno $(\mu \mathrm{g} / \mathrm{g})$ & $26,32 \pm 6,31$ \\
Carotenoides Totais $(\mu \mathrm{g} / \mathrm{g})$ & $10,36 \pm 0,49$ \\
atividade Antioxidante (ABTS) (mmol Trolox/g de & $3,06 \pm 0,99$ \\
Compostras $)$ & \\
\hline
\end{tabular}

Médias \pm desvio padrão. Fonte: Autores.

Carvalho et al. (2005) fizeram a estimativa indireta do teor de licopeno em frutos de genótipos de tomateiro via análise colorimétrica e identificaram o teor de licopeno no intervalo de 16 a $32 \mu \mathrm{g} / \mathrm{g}$ correspondendo ao estágio de maturação rosaesverdeado. O teor encontrado neste trabalho foi de $26,32 \pm 6,31 \mu \mathrm{g} / \mathrm{g}$, valor próximo ao encontrado no trabalho citado anteriormente.

A atividade antioxidante encontrada para a polpa concentrada foi de 3,06 $\pm 0,99 \mathrm{mMol}$ Trolox/g de amostra, já para o teor de compostos fenólicos foi obtido 347,40 \pm 144,00 mg EAG/100g. Vallverdú-Queralt (2012) avaliaram a atividade antioxidante e teor de fenólicos totais da polpa de tomate após a inativação enzimática ("hot break") e obtiveram através do método ABTS uma capacidade antioxidante 0,43 $\pm 0,21 \mathrm{mMol}$ Trolox/g de amostra e teor de fenólicos totais de 246,00 $\pm 9,1$ 
mg EAG/100g de amostra, teores menores do que os encontrados no presente trabalho mas que deve-se a influência da sazonalidade, espécie do tomate e ponto de maturação a qual cada um se encontrava.

\subsection{Análises físicas das cápsulas}

O peso e diâmetro das microcápsulas estão expressos na tabela 3. Baker (1986) definiu que as cápsulas podem ser classificadas por tamanho em 3 categorias: macro- $(>5000 \mu \mathrm{m})$, micro- $(0,2-5000 \mu \mathrm{m})$ e nanocápsulas $(<0,2 \mu \mathrm{m})$, assim, de acordo com essa classificação as cápsulas obtidas neste trabalho podem ser classificadas como microcápsulas.

Tabela 3: Tamanho e peso das microcápsulas M2 e M5.

\begin{tabular}{ccc}
\hline & M2 & M5 \\
\hline Peso $(\mathrm{g})$ & $0,11^{\mathrm{a}}$ & $0,07^{\mathrm{b}}$ \\
Diâmetro $(\mu \mathrm{m})$ & $4880,0^{\mathrm{a}}$ & $4520,0^{\mathrm{b}}$ \\
\hline
\end{tabular}

Médias seguidas pela mesma letra, na mesma linha, não diferem estatisticamente pelo teste de Tukey a 5\% de probabilidade. Fonte: Autores, (2020).

Houve diferença significativa $(\mathrm{p}<0,05)$ entre M2 e M5 para os dois parâmetros avaliados, como podemos observar na Tabela 3. Apesar dessa diferença não ter sido observada para a densidade (Tabela 1), uma pequena variação desse parâmetro contribuiu para formação de microcápsulas maiores e mais pesadas na formulação M2. A formulação M5 apresentou valores inferiores devido ao maior teor de alginato, que ocasionou a criação de espaços livres com ar no interior da cápsula, diminuindo assim o peso e diâmetro desta.

\subsection{Interação entre os compostos bioativos encapsulados e o azeite de oliva:}

Como pode ser observado na Figura 1, não foi observado interação entre os teores de licopeno da microcápsula e o azeite durante os dois meses de armazenamento, as microcápsulas conseguiram reter e conservar o licopeno. Silva et al. (2013) encapsularam licopeno com um complexo de gelatina-pectina através do método de coacervação e observaram que houve uma degradação de $14 \%$ do licopeno por semana.

Figura 1: Teor de licopeno $(\mu \mathrm{g} / \mathrm{g})$ das formulações M2 e do azeite A2 (Figura 1.A) e da microcápsula M5 e do azeite A5 (Figura 1.B)

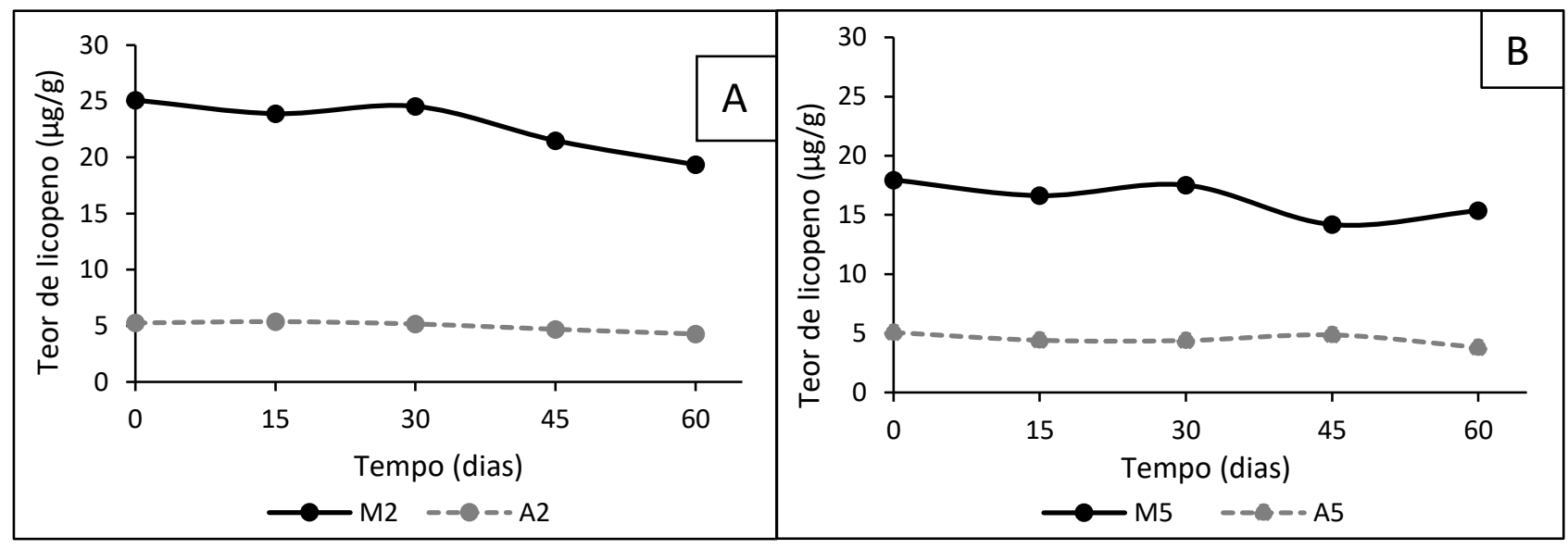

Fonte: Autores, (2020). 
Neste trabalho a taxa de degradação foi de aproximadamente 3\% por semana, uma taxa de degradação menor do que a técnica de coacervação citada anteriormente. De acordo com Rodriguez-Amaya (2002), a maior causa de degradação do licopeno durante o processamento e estocagem é a oxidação enzimática e não enzimática. A isomerização dos carotenoides trans para o isômero cis descolore o alimento e isso é promovido pelo contato com ácidos, tratamento térmico e exposição à luz. Os teores de fenólicos totais podem ser visualizados na Figura 2.

Figura 2: Teor de fenólicos totais da microcápsula M2 e do azeite A2 (Figura 2.A) e da microcápsula M5 e do azeite A5 (Figura 2.B).

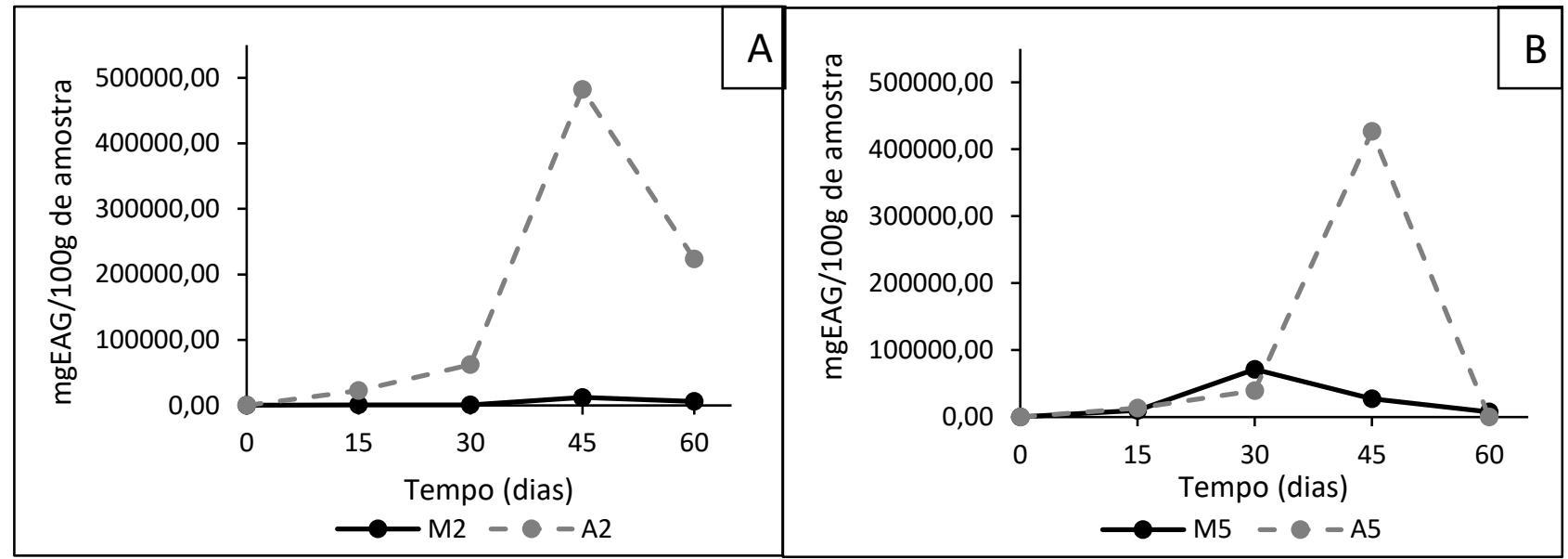

Fonte: Autores, (2020).

Como podemos observar nas figuras 2A e 2B o ponto mensurado nos 45 dias obteve uma leitura de grande elevação dos compostos fenólicos, provavelmente isso ocorreu devido à turbidez do produto e a interação com o reagente de FolinCiocalteu, pois segundo Waterhouse (2002) os produtos da reação que promovem a coloração azul apresentam alta absorção de luz, sendo que a intensidade da absorção de luz neste comprimento de onda é proporcional à concentração de compostos fenólicos. Desta forma, a turbidez gerada pode ter interferido na leitura real neste ponto. Em relação aos outros pontos analisados foi observado uma interação entre os compostos fenólicos das cápsulas em relação ao azeite de oliva extra-virgem. A composição M2 e A2 (azeite que foi drenado de AM2) se mantiveram mais estável, já a composição M5 e A5 (azeite que foi drenado de AM5) apresentaram uma maior interação e proporcionalidade, pois à medida que o teor de compostos fenólicos da cápsula aumentou o teor do azeite diminui, levando a observar uma possível existência de migração de um meio para o outro.

O comportamento da atividade antioxidante das microcápsulas M2 e M5 e dos seus respectivos azeites (A2 e A5) durante os 60 dias de armazenamento podem ser visualizados na Figura 3. 
Figura 3: Atividade antioxidante da microcápsula M2 e do azeite A2 (Figura 3.A) e da microcápsula M5 e do azeite A5 (Figura 3.B)

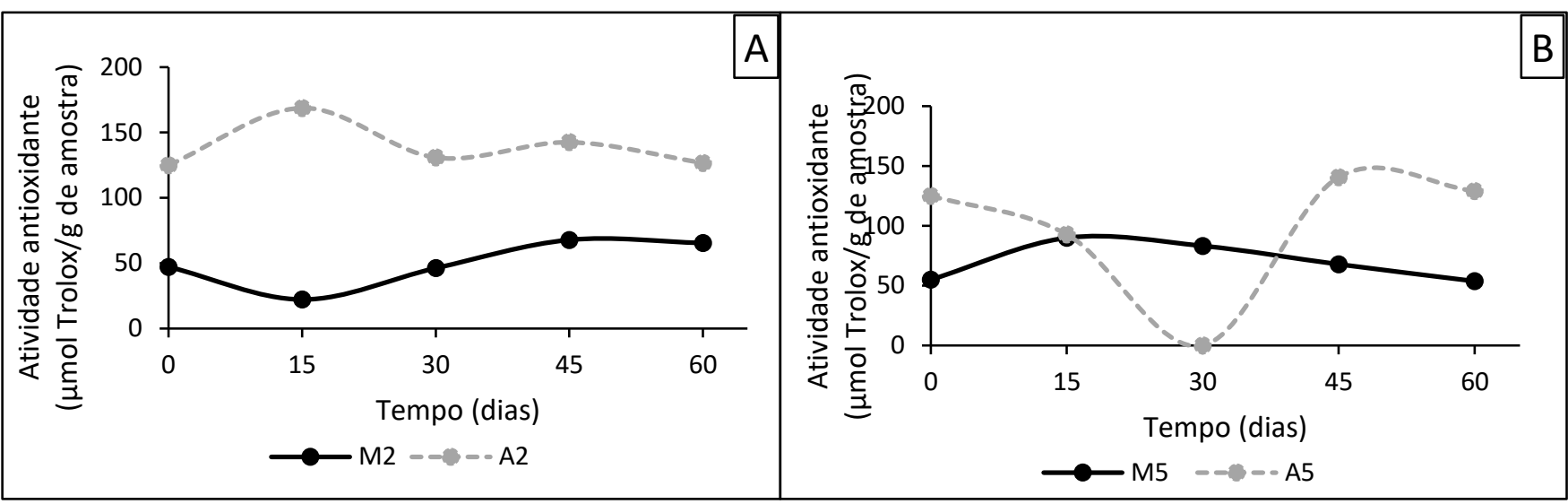

Fonte: Autores, (2020).

Pode ser observado na Figura 3A um aumento da atividade antioxidante do azeite proporcional à diminuição da atividade antioxidante das cápsulas M2 até o $15^{\circ}$ dia de armazenamento, após esse tempo verifica-se a estabilidade da amostra. A formulação M5 com o azeite A5 obteve uma interação maior em relação à atividade antioxidante, e como podemos observar houve uma proporcionalidade entre o aumento da atividade antioxidante das cápsulas e diminuição do azeite. O esperado seria que a formulação M5 com maior porcentagem de alginato obtivesse menor interação com o meio por possuir ligações mais fortes, porém a adição de água na proporção 1:3 (polpa de tomate:volume de água) pode ter gerado redes de alginato de cálcio mais fracas, facilitando a difusão com o meio.

A variação de carotenoides das microcápsulas M2 e M5 e dos seus respectivos azeites durante os 60 dias de armazenamento podem ser visualizados na Figura 4.

Figura 4: Variação do teor de carotenoides totais das microcápsulas das da microcápsula M2 e do azeite A2 (Figura 4.A) e da microcápsula M5 e do azeite A5 (Figura 4.B).

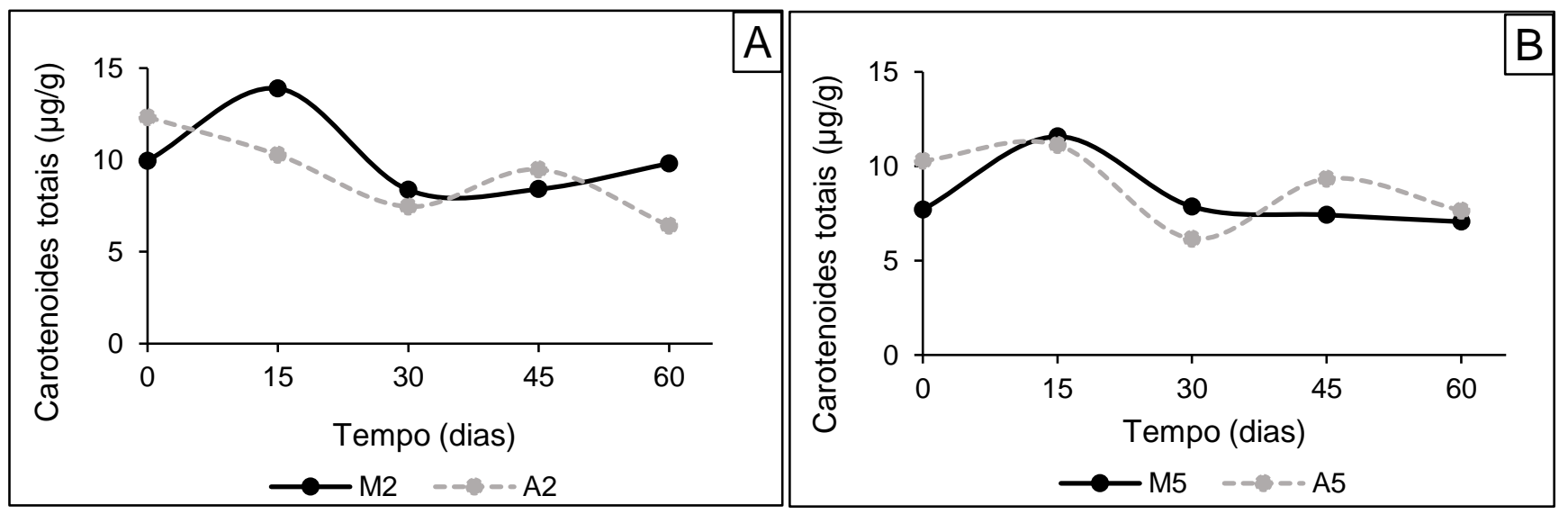

Fonte: Autores, (2020).

A formulação a M5 apresentou maior tendência ao equilíbrio a partir do $15^{\circ}$ dia de armazenamento, como pode ser observado na figura 4. De forma similar ao que aconteceu com o licopeno, o teor de carotenoides das microcápsulas M2 e M5 não apresentou oscilações durante o armazenamento, permanecendo estável, sendo assim um indicativo que a microcápsula conseguiu aprisionar e manter o teor de carotenoides, mesmo em presença de luz, que é uma das causas de deterioração. A 
formulação M2 obteve um valor final próximo ao encontrado neste trabalho com a polpa de tomate concentrada $(10,36 \pm 0,49$ $\mu \mathrm{g} / \mathrm{g}$ ), portanto, mesmo com o processo de gelificação o teor de carotenoides totais se manteve próximo ao encapsulado. $\mathrm{Na}$ formulação do azeite A2 foi observada uma diferença significativa ao nível de 5\% de probabilidade no teor de carotenoides totais durante o armazenamento, o azeite A5 se manteve estável e sem diferença significativa. No azeite de oliva virgem normalmente são relatados teores de carotenoides de 1 a $10 \mu \mathrm{g} / \mathrm{g}$ (Mínguez-Mosquera; Garrido-Fernandez, 1989). Apesar da diminuição do teor de carotenoides, que pode ter sido ocasionada por meio da degradação destes componentes em presença de luz, os teores se mantiveram dentro do intervalo esperado para o azeite de oliva extra virgem.

\subsection{Influência da cápsula na qualidade do azeite}

Nas tabelas 4 e 5 constam os resultados das análises de índice de peróxido e estabilidade oxidativa, respectivamente, parâmetros de suma importância para se avaliar a qualidade do azeite durante o armazenamento.

Tabela 4: Índice de peróxido (meq/kg) dos azeites de oliva extra virgem A2 e A5.

\begin{tabular}{cccccc}
\hline \multirow{2}{*}{ Amostra } & \multicolumn{5}{c}{ Tempo (dias) } \\
& 0 & 15 & 30 & 45 & 60 \\
\cline { 2 - 6 } A2 & $5,80^{\mathrm{d}}$ & $9,75^{\mathrm{c}}$ & $12,38^{\mathrm{b}}$ & $13,45^{\mathrm{b}}$ & $16,44^{\mathrm{a}}$ \\
A5 & $5,81^{\mathrm{d}}$ & $9,74^{\mathrm{c}}$ & $13,38^{\mathrm{b}}$ & $15,35^{\mathrm{b}}$ & $16,25^{\mathrm{a}}$ \\
\hline
\end{tabular}

Médias seguidas pela mesma letra, na mesma linha, não diferem estatisticamente pelo teste de Tukey a 5\% de probabilidade Fonte: Autores, (2020).

A análise de índice de peróxido foi realizada apenas no azeite drenado das microcápsulas, os resultados podem ser visualizados na tabela 4. As formulações analisadas diferiram entre si durante o armazenamento ao nível de 5\% de probabilidade, houve uma uniformidade no índice de peróxido durante o tempo de avaliação. As duas formulações tiveram um comportamento semelhante durante os dois meses de acompanhamento, assim independente da formulação de microcápsula (M2 e M5) adicionada aos azeites A2 e A5, não houve uma mudança da sua qualidade. No decorrer do armazenamento houve um aumento do índice de peróxido, indicando assim um aumento no grau de oxidação do azeite, mesmo com esse fenômeno após os 60 dias de armazenamento as formulações dos azeites obtiveram um índice de peróxido dentro do permitido para comercialização designado pela legislação como sendo menor do que $20 \mathrm{meq} / \mathrm{kg}$ (BRASIL,2012). Os valores obtidos neste trabalho foram próximos aos encontrados por Santos (2010), que avaliou o índice de peróxido de azeites aromatizados com tangerina, laranja e limão, ele obteve os seguintes valores 16,48, 16,16 e 16,34 meq $/ \mathrm{kg}$, respectivamente. Assim mesmo adicionando outro alimento ao azeite, para uma composição diferente, este fator não diminuiu a qualidade do azeite de oliva extra virgem, ainda permanecendo dentro do limite exigido pela legislação.

A estabilidade oxidativa, determinada no Rancimat, foi realizada no conjunto azeite + microcápsula (AM2- azeite com a formulação das microcápsulas a 2\%; AM5- azeite com a formulação das microcápsulas a 5\%) (Tabela 7).

Tabela 5: Período de indução (horas) da análise de estabilidade oxidativa das formulações AM2 e AM5.

\begin{tabular}{cccccc}
\hline Amostra & \multicolumn{5}{c}{ Tempo (dias) } \\
& 0 & 15 & 30 & 45 & 60 \\
\cline { 2 - 6 } AM2 & $5,93^{\mathrm{a}}$ & $6,02^{\mathrm{a}}$ & $6,80^{\mathrm{a}}$ & $7,38^{\mathrm{a}}$ & $6,03^{\mathrm{a}}$ \\
AM5 & $5,93^{\mathrm{a}}$ & $6,93^{\mathrm{a}}$ & $6,48^{\mathrm{a}}$ & $6,64^{\mathrm{a}}$ & $6,94^{\mathrm{a}}$ \\
\hline
\end{tabular}

Médias seguidas pela mesma letra, na mesma linha, não diferem estatisticamente pelo teste de Tukey a 5\% de probabilidade. Fonte: Autores, (2020). 
O azeite oliva extra virgem com microcápsulas de tomate obteve um período de indução de 6,1 $\pm 0,23$ horas. Jorge (2010) avaliou a estabilidade oxidativa de alguns azeites extra virgem da região de Pelotas através do equipamento Rancimat utilizando as condições de $110^{\circ} \mathrm{C}$ e $10 \mathrm{~L} / \mathrm{h}$ de fluxo de ar e obteve um tempo entre 9 à 15 horas do período de indução. Apesar do resultado do trabalho ter sido inferior ao trabalho citado anteriormente, devemos levar em consideração a origem do azeite, sazonalidade e outros fatores que diferem as condições e tempo de oxidação de cada tipo de azeite de oliva extra virgem. As formulações AM2 e AM5 não diferiram significativamente durante o tempo de armazenamento, como apresentado na tabela 5. Houve um aumento do período de indução das duas formulações analisadas, a formulação AM5 obteve um período de indução maior do que o período obtido no azeite de oliva extra virgem (6,1 $\pm 0,23$ horas), sem as microcápsulas. Desta forma a junção das microcápsulas com o azeite de oliva contribui de forma positiva para a qualidade de um novo pronto, pois agrega os compostos nutricionais gerando assim um produto mais nutritivo e mais resistente a oxidação lipídica, mesmo sendo armazenado em exposição à luz.

\subsection{Análise de DSC}

Como podemos ver nas figuras 5 e 6 foi observado um comportamento endotérmico que variou a sua temperatura de pico entre 100 e $109^{\circ} \mathrm{C}$. Segundo Smitha, Sridhar e Khan (2005) que estudaram a estabilidade térmica dos filmes alginato de sódio-quitosana a faixa entre $110^{\circ} \mathrm{C}$ e $200^{\circ} \mathrm{C}$, são resultantes da eliminação da água e da formação de ligações amidas na estrutura do íon complexo. Laia (2015) também encontrou uma faixa entre 30 e $200^{\circ} \mathrm{C}$ um evento endotérmico, caracterizando a desidratação da amostra, quando estudou a estabilidade térmica do alginato de sódio em pó. O segundo evento encontrado foi um comportamento exotérmico que ocorreu em uma faixa de temperatura de pico entre 390 e $400^{\circ} \mathrm{C}$, que é característico da degradação térmica do alginato de sódio.

Laia (2015) analisou a estabilidade térmica do alginato de sódio em pó e obteve em uma faixa de 200 a $550{ }^{\circ} \mathrm{C}$ um evento exotérmico que caracterizava a degradação do alginato de sódio. As formulações M2 (Figura 5) e M5 (Figura 6) obtiveram os seus picos de desidratação e degradação em uma mesma faixa, tendo assim características térmicas semelhantes. Conhecer os pontos de desidratação e degradação das microcápsulas ajuda a entender o comportamento destas frente a condições de armazenamento ou processamento que podem sofrer, ajudando assim a delimitar os parâmetros para o controle de qualidade. 
Figura 5: $\mathrm{M} 2 / \mathrm{A}=0 \mathrm{~h}, \mathrm{~B}=15$ dias; $\mathrm{C}=30$ dias; $\mathrm{D}=45 \mathrm{dias} ; \mathrm{E}=60 \mathrm{dias}$.

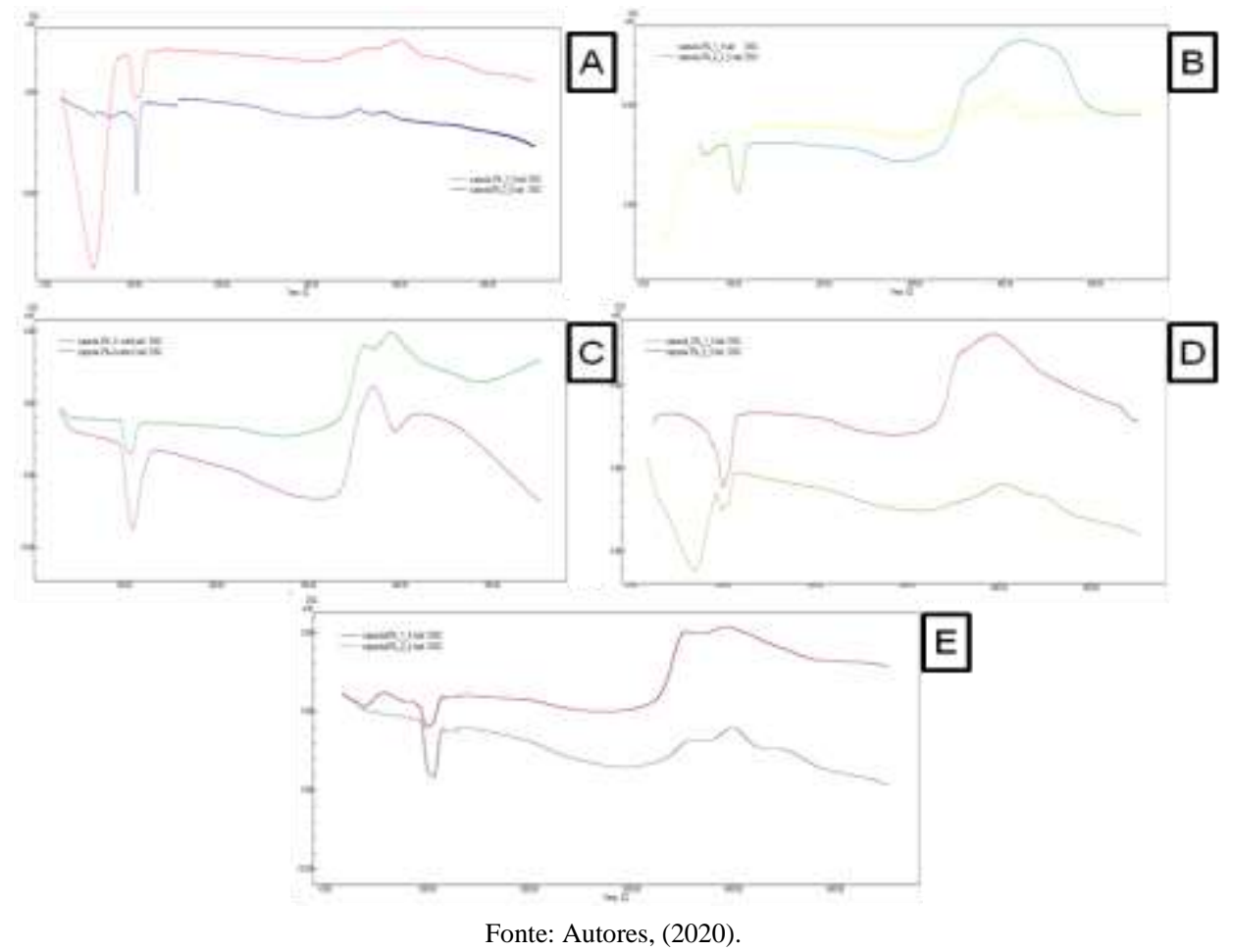

Figura 6: $\mathrm{M} 5$ / $\mathrm{A}=0 \mathrm{~h}, \mathrm{~B}=15$ dias; $\mathrm{C}=30$ dias; $\mathrm{D}=45$ dias; $\mathrm{E}=60$ dias.

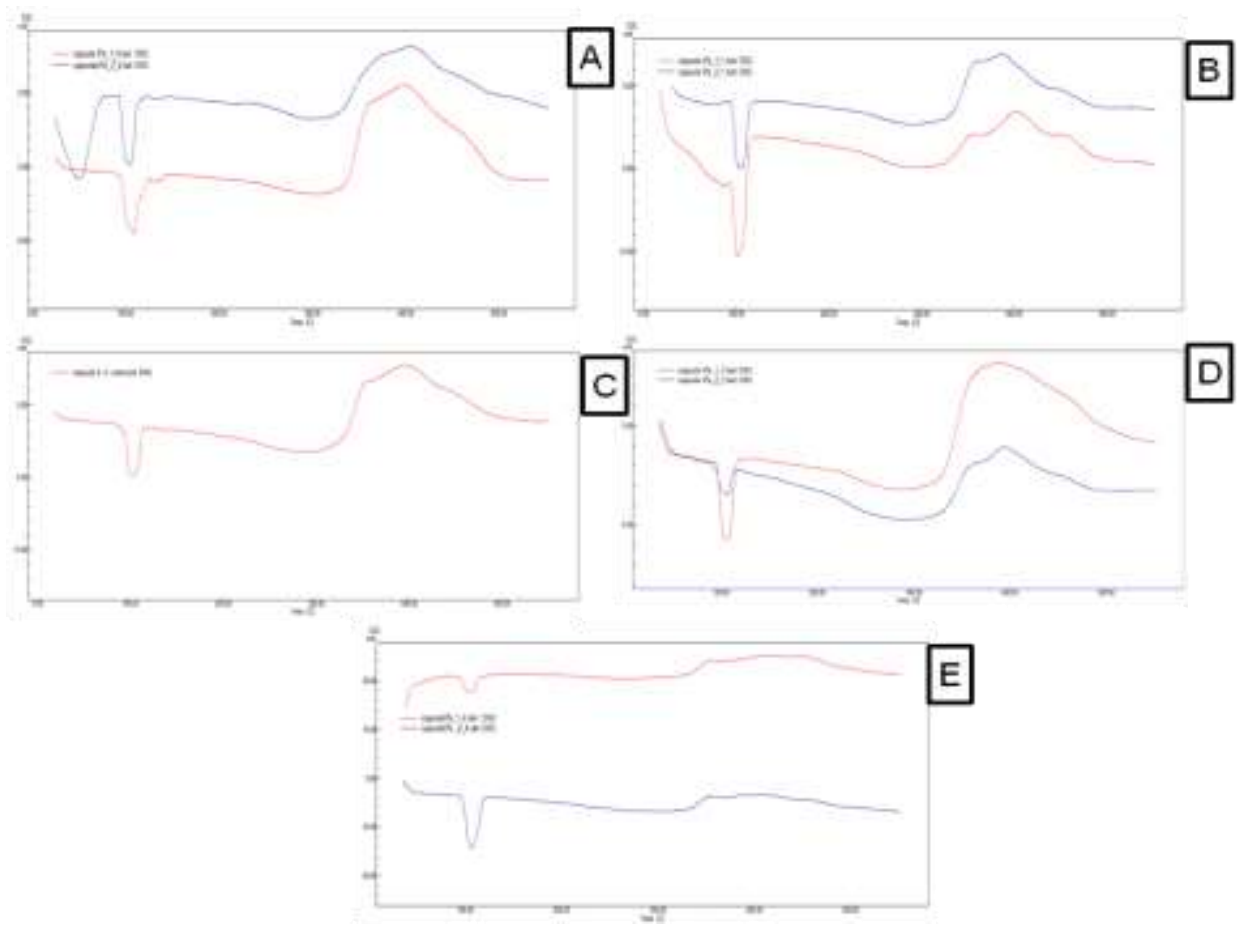

Fonte: Autores, (2020). 


\section{Conclusão}

As formulações M2 e M5 apresentaram desempenho semelhante no quesito estabilidade de armazenamento para o teor de licopeno e carotenoides, já para o teor de fenólicos totais e atividade antioxidante a formulação M2 obteve uma maior estabilidade e a formulação M5 obteve maior difusão do encapsulado em relação ao meio. A adição das microcápsulas no azeite de oliva extra-virgem não alterou a sua qualidade em relação ao índice de peróxido e estabilidade oxidativa. Desta forma pode-se concluir que a formulação M2 obteve um desempenho eficiente em aprisionar o encapsulado, já a formulação M5 pode ser utilizada com o objetivo de difusão controlada do encapsulado em um meio e a adição destas em um meio oleoso não interferirá em sua qualidade. Assim, diferentes concentrações de encapsulados e encapsulantes poderiam ser estudadas em trabalhos futuros, visando obter um produto com características de aprisionamento ou liberação de acordo com o objetivo, levando mais qualidade ao consumidor.

\section{Referências}

AOAC: Association of Official Analytical Chemists.(2003). Official methods of analysis of the Association of Official Analytical Chemists. $5^{\text {a }}$ ed. Arlington: A.O.A.C., 2003.

Baker, R. W. (1987). Controlled release of biologically active agents. New York: John Wiley \& Sons

Boroski, M.; Visentainer, J. V.; Cottica, S. M. \& Morais, D. R. (2015). Antioxidantes: princípios e métodos analíticos. Appris.

Brasil.(2005). Agência Nacional de Vigilância Sanitária. Resolução-rdc n ${ }^{\circ} 272$, de 22 de setembro de 2005. "Regulamento técnico para produtos de vegetais, produtos de frutas e cogumelos comestíveis”. Diário Oficial da União; Poder Executivo, 2005.

Brasil.(2012). Instrução Normativa $\mathrm{n}^{\circ}$ 1, de 30 de janeiro de 2012. Regulamento Técnico do Azeite de Oliva e do Óleo de Bagaço de Oliva. Diário oficial da União: seção 1, Brasília, DF, n 23, p. 5, 01 de fev. 2012.

Carmo, E. L. D., de Barros Fernandes, R. V. \& Borges, S. V. (2015). Microencapsulação por spray drying, novos biopolímeros e aplicações na tecnologia de alimentos. The Journal of Engineering and Exact Sciences, 1(2), 30-44.

Carvalho, W., Fonseca, M. E. D. N., Silva, H. R. D., Boiteux, L. S. \& Giordano, L. D. B. (2005). Estimativa indireta de teores de licopeno em frutos de genótipos de tomateiro via análise colorimétrica. Horticultura Brasileira, 23(3), 819-825.

Choudhari, S., Bajaj, I., Singhal, R. \& Karwe, M. (2012). Microencapsulated lycopene for pre-extrusion coloring of foods. Journal of Food Process Engineering, 35(1), 91-103.

Clinton, S. K. (1998). Lycopene: chemistry, biology, and implications for human health and disease. Nutrition Reviews, v. 56, n. 2 Pt 1, p. 35-51.

Costa-Rodrigues, J., Pinho, O. \& Monteiro, P. R. R. (2018). Can lycopene be considered an effective protection against cardiovascular disease?. Food chemistry, 245, 1148-1153.

Di Mascio, P., Murphy, M. E. \& Sies, H. (1991). Antioxidant defense systems: the role of carotenoids, tocopherols, and thiols. The American journal of clinical nutrition, 53(1), 194S-200S

Fang, Z. \& Bhandari, B. (2010). Encapsulation of polyphenols-a review. Trends in Food Science \& Technology, 21 (10), 510-523.

Fávaro-Trindade, C. S. Pinho, S., Rocha, G. A. (2008). Revisão: encapsulação de ingredientes alimentícios. Brazilian Journal of Food Technology, v.11, n. 2, p.103-112.

Goula, A. M. \& Adamopoulos, K. G. (2012). A new technique for spray-dried encapsulation of lycopene. Drying Technology, 30(6), 641-652.

Ha, T. V. A., Kim, S., Choi, Y., Kwak, H. S., Lee, S. J.; Wen, J. \& Ko, S. (2015). Antioxidant activity and bioaccessibility of size-different nanoemulsions for lycopene-enriched tomato extract. Food chemistry, 178, 115-121.

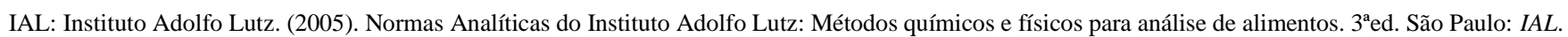

Jorge, R. O. (2010). Caracterização de azeites virgem extra gourmet varietais e blends comercializados no mercado do Rio Grande do Sul. Tese de doutorado, Programa de pós-graduação em Ciência e Tecnologia Agroindustrial, Faculdade de Agronomia Eliseu Maciel. Rio Grande do Sul, Brasil.

Laia, A. G. S. (2015). Estudo de filmes e hidrogéis a base de alginato e goma gelana visando aplicações na regeneração de discos intervertebrais. Dissertação de Mestrado, Programa de pós-graduação em Engenharia de Materiais, Instituto Federal de Minas Gerais, Minas Gerais, Brasil.

Lichtenthaler, H. K. (1987). Clorofilas e carotenóides: pigmentos de biomembranas fotossintéticas. Methods in enzymology , 148 , $350-382$.

Mirzaei, H.; Pourjafar, H. \& Homayouni, A. (2012). Effect of calcium alginate and resistant starch microencapsulation on the survival rate of Lactobacillus acidophilus La and sensory properties in Iranian white brined cheese. Food Chemistry, 132(4), 1966-1970. 
Palozza, P. A. O. L. A., Catalano, A., Simone, R. E., Mele, M. C. \& Cittadini, A. (2012). Effect of lycopene and tomato products on cholesterol metabolism. Annals of Nutrition and Metabolism, 61(2), 126-134.

Passos, R.M., Santos, J., Silva, G.F., Pagani, A.A.C.(2021). Azeite de oliva com pérolas de tomate: avaliação sensorial, microbiológica e estabilidade da cor. Revista Brasileira de Agrotecnologia - ISSN 2317-3114 - (BRASIL) v. 11, n.2, p.498-504.

Ranveer, R. C., Gatade, A. A., Kamble, H. A. \& Sahoo, A. K. (2015). Microencapsulation and storage stability of lycopene extracted from tomato processing waste. Brazilian archives of biology and technology, 58(6), 953-960.

Rocha, G. A.; Fávaro-Trindade, C. S. \& Grosso, C. R. F. (2012). Microencapsulation of lycopene by spray drying: characterization, stability and application of microcapsules. Food and bioproducts processing, 90(1), 37-42.

Rocha, L. C. R. (2017). Desenvolvimento de micropartículas contendo suco de tomate via gelificação iônica. Dissertação de mestrado, Programa de pósgraduação em Engenharia de Biomateriais, Universidade Federal de Lavras. Minas Gerais, Brasil.

Rodriguez-Amaya, D. B. (2001). A guide to carotenoid analysis in foods. ILSI Human Nutrition Institute, v. 64, p. $20005-5802$.

Rodriguez-Amaya, D. B. (2002). Effects of processing and storage on food carotenoids. Sight and Life Newsletter, 3(Special Issue), 25-35.

Rodrigues, J. F. (2015). Azeites de oliva da região da Serra da Mantiqueira: estudo químico e sensorial para caracterização da qualidade. Dissertação de mestrado, Programa de pós-graduação em ciência de Alimentos, Universidade Federal de Lavras. Minas Gerais, Brasil.

Santos, S. I. F. (2010). Desenvolvimento de um azeite com aroma a limão. Dissertação de mestrado, Programa de pós-graduação em Biotecnologia, Universidade de Aveiro, Aveiro, Portugal.

Silva, F. D. A. S. \& de Azevedo, C. A. V. (2009). Principal Components Analysis in the Software Assistat-Statistical Assistance. In 7th World Congress on Computers in Agriculture Conference Proceedings, 22-24 June 2009, Reno, Nevada (p. 1). American Society of Agricultural and Biological Engineers.

Silva, F. C., da Fonseca, C. R., de Alencar, S. M., Thomazini, M., de Carvalho Balieiro, J. C., Pittia, P. \& Favaro-Trindade, C. S. (2013). Assessment of production efficiency, physicochemical properties and storage stability of spray-dried propolis, a natural food additive, using gum Arabic and OSA starchbased carrier systems. Food and Bioproducts Processing, 91(1), 28-36.

Smitha, B., Sridhar, S. \& Khan, A. A. (2005). Chitosan-sodium alginate polyion complexes as fuel cell membranes. European Polymer Journal, 41(8), 18591866

Swain, T. \& Hillis, W. E. (1959). The phenolic constituents of Prunus domestica. I.-The quantitative analysis of phenolic constituents. Journal of the Science of Food and A griculture, 10(1), 63-68.

Vallverdú-Queralt, A., Medina-Remón, A., Casals-Ribes, I., Andres-Lacueva, C., Waterhouse, A. L. \& Lamuela-Raventos, R. M. (2012). Effect of tomato industrial processing on phenolic profile and hydrophilic antioxidant capacity. LWT-Food Science and Technology, 47(1), 154-160.

Waterhouse, A. L. (2002). Determination of total phenolics. Current protocols in food analytical chemistry, 6(1), I1-1.

Zu, K., Mucci, L., Rosner, B. A., Clinton, S. K., Loda, M., Stampfer, M. J. \& Giovannucci, E. (2014). Dietary lycopene, angiogenesis, and prostate cancer: a prospective study in the prostate-specific antigen era. Journal of the National Cancer Institute, 106(2), djt430. 